J. Clin. Chem. Clin. Biochem.

Vol. 18, 1980, pp. 797-805

\title{
Der Einsatz der Lasernephelometrie zur Bestimmung und rechnerunterstützten Auswertung der Fibronectinkonzentration in verschiedenen Körperflüssigkeiten
}

\author{
Von A. M. Gressner und P. Wallraff \\ Abteilung Klinische Chemie und Pathobiochemie, Klinisch-Chemisches Zentrallaboratorium \\ der Medizinischen Fakultät der R.WTH, Aachen
}

(Eingegangen am 3. März/2. Juli 1980)

Zusammenfassung: Es wird über eine lasernephelometrische Methode zur Bestimmung der Fibronectinkonzentration in verschiedenen menschlichen und tierischen Körperflüssigkeiten berichtet. Das Verfahren erweist sich als präzise (VK in der Serie 2,1\%), empfindlich (untere Nachweisgrenze 1,2 mg/l Fibronectin), schnell, relativ einfach und voll mechanisierbar. Mittels programmierbarer Rechner ist eine elektronische Auswertung der Streulichtsignale möglich. Die mit der Lasernephelometrie und der radialen Immundiffusion bestimmten Antigenkonzentrationen weisen einen Korrelationskoeffizienten von $\mathrm{r}=0,859$ auf.

In Plasmen gesunder Männer wurde eine mittlere Fibronectinkonzentration von $291 \mathrm{mg} / \mathrm{l}$, bei Frauen eine signifikant niedrigere Konzentration von $259 \mathrm{mg} / 1$ gefunden. Bei Carcinomen des weiblichen Genitaltraktes und bei Morbus Crohn lassen sich Erhöhungen der Fibronectinkonzentration im Plasma feststellen. Synovialflüssigkeiten entzündlicher Genese weisen signifikant höhere Konzentrationen $(318 \mathrm{mg} / \mathrm{l})$ auf als die nicht-entzündlichen Ursprungs. In Seminalplasmen konnte bei starker individueller Streuung Fibronectin in dem Plasma vergleichbaren Konzentrationen nachgewiesen werden. Extrem niedrige Antigenkonzentrationen $(0,41 \mathrm{mg} / \mathrm{l})$ fanden sich im Liquor cerebrospinalis Gesunder, unter pathologischen Bedingungen kommt es zu signifikanten, positiv mit der Zellzahl korrelierenden Anstiegen. Ebenfalls niedrige Fibronectinkonzentrationen $(16 \mathrm{mg} / 1)$ sind in Amnionflüssigkeit am Ende der Gravidität nachweisbar.

The use of laser nephelometry for the determination and computer-assisted calculation of the fibronectin concentration in various body fluids

Summary: We report a laser-nephelometric procedure for the determination of the concentration of fibronectin in various human and animal body fluids. The method is precise (CV intra-assay $2.1 \%$ ), sensitive (the detection limit is $1.2 \mathrm{mg} / \mathrm{l}$ fibronectin), rapid, relatively simple and can be totally mechanized. By use of programmable calculators an electronic evaluation of the scattered light signals is possible. The antigen concentrations determined both by laser nephelometry and radial immunodiffusion show a coefficient of correlation of $r=0.859$.

In plasma of healthy men the mean concentration of fibronectin is $291 \mathrm{mg} / \mathrm{l}$; women have a significant lower concentration of $259 \mathrm{mg} / \mathrm{l}$. Increased levels of plasma fibronectin were found in patients with carcinomas of the female genital tract and Morbus Crohn. Synovial fluids obtained from inflammatory joint diseases contain significantly higher concentrations of fibronectin $(318 \mathrm{mg} / \mathrm{l})$ than those from non-inflammatory joints. The range of concentration of fibronectin in seminal plasma is similar to that in blood plasma, but a large inter-individual variation was observed. Extremely low antigen concentrations $(0.41 \mathrm{mg} / \mathrm{l})$ were determined in normal liquor cerebrospinalis, but under pathological conditions a significant increase occurs, which correlates strongly with the cell count. Low levels of fibronectin $(16 \mathrm{mg} / \mathrm{l})$ were also found in amniotic fluids at the end of pregnancy.

\section{Einführüng}

Fibronectin (1), auch unter den Bezeichnungen fibroblast surface antigen (2), galactoprotein $A(3)$, cell surface protein (4), large, external, transformation-sensitive (LETS) protein (5) und cold insoluble globulin (6) bekannt, kommt als hochmolekulares Glykoprotein an den Oberflächen vorwiegend mesenchymaler Zellen als Bestandteil der Glycocalyx, im extrazellulären Bindegewebe und in extrazellulären Flüssigkeiten vor (für Ubersichten siehe 1. c. 7-9). Im Plasma tritt es als dimeres, aus zwei nahezu identischen Untereinheiten bestehendes und durch Disulfidbrücken fixiertes Protein mit einem Molekulargewicht von $\mathbf{4 4 0 0 0 0}$ und elektro- 
phoretischer $\alpha_{2}$-Beweglichkeit auf (10), welches der zellassoziierten Form des Fibronectins zwar sehr ähnlich, mit ihr wahrscheinlich jedoch nicht identisch ist (7). Immunologisch zeichnen sich beide Typen durch komplette Kreuzreaktivität aus (2,11-13).

Die Vielzahl der biologischen Aktivitäten dieses Proteins, wie seine Bindungsaffinität zu Kollagen (14-16), Fibrin und Fibrinogen (17), sein positiver Effekt auf die Zell-Zell- und Zell-Substrat-Adhäsion $(18,19)$, seine Hämagglutinationseigenschaften $(20,21)$ und Opsoninaktivität (22), seine vermutete Rolle in der ClearanceFunktion des retikuloendothelialen Systems (23) und seine mögliche Bedeutung bei embryonalen Differenzierungsprozessen (24), sowie die Verminderung des oberflächenassoziierten Fibronectins in transformierten (neoplastischen) Zellen $(25,26)$ haben das klinische Interesse an diesem Plasmaprotein stark ausgeweitet. Damit entstand der Bedarf an einer schnellen, einfachen und zuverlässigen Methode zur quantitativen Bestimmung des Fibronectins im Plasma und in Geweben.

Im Gegensatz zu den früher verwendeten, bestenfalls semiquantitativen Nachweisverfahren durch Immunfluoreszenz oder durch elektrophoretische Auftrennung mit nachfolgender Densitometrie haben sich immunologische Meßprinzipien, wie radiale Immundiffusion (27), Elektroimmundiffusion nach Laurell (28), Radioimmunoassay $(1,29)$ und Enzymimmunoassay (ELISA) (15) als Methoden mit relativ hoher analytischer $\mathrm{Zu}$ verlässigkeit erwiesen. Der Einsatz der Lasernephelometrie, z.B. bei der quantitativen Bestimmung der Immunglobulinkonzentrationen im Serum $(30,31)$, hat gezeigt, daß sich dieses Verfahren grundsätzlich zur schnellen, einfachen und zuverlässigen Einzelproteinbestimmung anbietet $(32,33)$.

Wir haben dieses Prinzip folglich zur Bestimmung der Fibronectinkonzentration in verschiedenen menschlichen und tierischen Körperflüssigkeiten angewandt und an einigen Beispielen geprüft, ob sich aus veränderten Fibronectinkonzentrationen diagnostisch relevante Aussagen machen lassen.

\section{Material und Methoden}

Geräte

Als Grundgerät diente das mit einem $4 \mathrm{~mW}$ Helium-Neon-Laser (Wellenlänge 632,8 nm) ausgestattete Nephelometẹr (Nr. 37250/ 339) der Behringwerke AG, Marburg/Lahn (34), weiches durch eine Transportautomatik mit elektronischer Zeitvorwahl (Behringwerke $A G$ ) und durch einen über ein Interface adaptierten programmierbaren Hewlett-Packard-Tischrechner $9815 \mathrm{~A}$ ergänzt war. Die Streulichtintensitäten wurden bei manueller Auswertung an einem Digitalvoltmeter abgelesen oder bei elektronischer Auswertung unter Verwendung geeigneter Programme (siehe unten) direkt in die Endkonzentration umgerechnet und ausgedruckt. Die Inkubation und Streulichtmesșung erfolgte in LN-Universalküvetten (Behringwerke AG).

\section{Reagenzien}

Die Reagenzien wurden von folgenden Firmen bezogen: Antiserum gegen humanes Fibronectin vom Kaninchen $(0,79 \mathrm{~g}$ Protein/ml, Charge 107202 B, C), Protein-Standardplasma (Charge 1105, $250 \mathrm{mg}$ Fibronectin/l), LC Partigen-Platten für Fibronectin $^{1}$ ) (Charge 3674) und Klärungsmittel für trübe Sera (unter Verwendung von Frigen ${ }^{\circledR}$ ) von der Fa. Behring AG, Marburg/Lahn; Polyethylenglykol 6000 pract. von Serva Feinbiochemica, Heidelberg; phosphatgepufferte NaCl-Lösung (ohne Magnesium und Calcium) von Flow Laboratories GmbH, Bonn; humanes Fibronectin (Charge 93457, elektrophoretische Reinheit $85 \%, 0,8 \mathrm{~g} / 1$ Cyclohexylaminopropan-Sulfonsäure, $\mathrm{pH} 11,0$ ) von Collaborative Research, Inc., Waltham, Mass., USA; Hyaluronat 4-glycanohydrolase (EC 3.2.1.35) von Boehringer $\mathrm{GmbH}$, Mannheim; Heparin (Charge $78 \mathrm{C}-0203$, Na-Salz, aus Intestinalmucosa des Schweines) von Sigma Chemical Comp., München.

\section{Proben}

Die Fibronectinkonzentrationen wurden bestimmt in Plasmen offensichtlich (klinisch und biochemisch) gesunder Personen (Blutspender, Studenten, Mitarbeiter der Klinik) zur Erstellung des Referenzbereiches sowie in den in das Zentrallaboratorium gelangten, durch eindeutige Diagnosen ausgewiesenen pathologischen Plasmen. Die Proben wurden bis zur Analyse, die überwiegend innerhalb von 24 Stunden nach Specimennahme erfolgte, bei $4{ }^{\circ} \mathrm{C}$ gelagert.

Synovialflüssigkeit wurde durch Punktion ätiologisch unterschiedlicher Gelenkergüsse gewonnen und nach Abzentrifugation zellulärer Bestandteile bis zur weiteren Verwendung bei $-20^{\circ} \mathrm{C}$ gelagert. Unmittelbar vor der Analyse wurden die Proben durch Inkubation mit $25 \mu \mathrm{g}$ (ca. 25 Einheiten) Schaftestes-Hyaluronidase pro $\mathrm{ml}$ Synovialflüssigkeit für 5 Minuten bei $37^{\circ} \mathrm{C}$ in der früher beschriebenen Weise depolymerisiert (35).

Seminalplasma wurde aus dem zum Żwecke der Fertilitätsdiagnostik gewonnenen Ejakulat nach Abzentrifugation der Zellen hergestellt und ohne längere Probenlagerung direkt zur Untersuchung eingesetzt.

Die zur Fibronectinbestimmung verwendeten Volumina Amnion-flüssigkeit und Liquor cerebrospinalis waren jeweils ein Teil der zu anderen klinisch-diagnostischen Zwecken durch Amniozentese bzw. Lumbalpunktion abgenommenen Körperflüssigkeiten.

Zur Gewinnung von Rattenplasma wurden äthernarkotisierten männlichen Sprague-Dawley Ratten etwa $5 \mathrm{ml}$ Blut aus der V. cava inf. entnommen und in EDTA-Blutbildröhrchen antikoaguliert.

\section{Durchführung der Fibronectinbestimmung}

Mit Ausnahme der in den Legenden der entsprechenden Abbildungen und Tabellen beschriebenen Modifikationen war das grundsätzliche Vorgehen bei der lasernephelometrischen Bestimmung der Fibronectinkonzentration wie folgt:

2 bis $5 \mathrm{ml}$ Blut wurden unmittelbar nach Entnahme in Ethylendiamintetraacetat-(EDTA-) beschichteten Röhrchen (Fa. Sarstedt, Art. Nr. 328) antikoaguliert und zentrifugiert. $0,5 \mathrm{ml}$ des hämolysefreien Plasmas wurden mit Klärungsmittel behandelt $(36,37)$ und $100 \mu l$ des geklärten Plasmas 1:51 mit filtrierter (Millex disposable filters, Millipore $\mathrm{GmbH}$, Neu-Isenburg) $0,154 \mathrm{~mol} / \mathrm{l} \mathrm{NaCl}-L o ̈ s u n g ~ v e r d u ̈ n n t .100 \mu \mathrm{l}$ des verdünnten Plasmas wurden in LN-Küvetten, deren Eigenstreuung vorher geprüft wurde, mit $100 \mu 1$ des $1: 5$ verdünnten Fibronectin-Antiserums für genau $60 \mathrm{~min}$ bei Raumtemperatur inkubiert und zur Streulichtmessung eịngesetżt. Bei seriellen Bestimmungen erfolgte die Zugabe von Antiserum und Messung im 15 s Intervall.

Die Leerwerte (in Volt) waren für die Küvetten 0,04, für filtrierte $\mathrm{NaCl} 0,06$, für verdünntes Antiserum 0,22 und verdünntes, geklärtes Plasma 0,08.

Zur Erstellung der Referenzkurve wurden die Streulichtintensitäten geometrischer Verdünnungen von $1: 13$ bis $1: 208$ des

1) Kein offizielles Verkaufsprodukt der Behringwerke. 
geklärten Protein-Standardplasmas ( $250 \mathrm{mg} / 1$ Fibronectin) $\mathrm{mit}$ filtrierter phy siologischer $\mathrm{NaCl}$-Lösung gemessen und auf Millimeterpapier ausgewertet. Diese Kurve diente auch zur Grundlage der rechnerunterstütz ten Auswertung.

Zur Fibronectinbestimmung durch radiale Immundiffusion wurde geklärtes EDTA-Plasma 1:3 mit $0,154 \mathrm{~mol} / 1 \mathrm{NaCl}$ verdünnt. $20 \mu l$ dieser Verdünnung wurden aufgetragen und 3 Tage inkubiert. Die Standardkurve wurde mit unverdünntem $(250 \mathrm{mg} / \mathrm{l}), 1: 2(125 \mathrm{mg} / \mathrm{l})$ und $1: 4(62,5 \mathrm{mg} / \mathrm{l})$ verdünntem Standardhumanplasma (Charge 1105) erstellt. Die Auswertung erfolgte nach dem üblichen Verfahren.

\section{Allgem eine Analysen}

Die Bestimmung der Proteinkonzentrationen wurde mit der Biuret-Methode (38), Zellzählungen und -differenzierungen nach klinisch-chemischen Standardverfahren durchgeführt.

\section{Statistische Verfahren}

Zur Ermittlung des Referenzbereiches wurde das vom Verteilungstyp der Meßwerte unabhängige nicht-parametrische Testverfahren nach Murphy (39) angewandt. Signifikanzen wurden geprüft entweder mit dem t-Test für unverbundene und verbundene Stichproben oder mit dem Wilcoxon-Test (40).

\section{Ergebnisse}

\section{Methodik}

Die Inkubation einer Plasmaverdünnung mit FibronectinAntiserum führt zu einer zeitabhängigen Zunahme der Intensität des gestreuten Laserlichtes (Abb. 1). Nahezu maximale Streulichtsignale werden nach einer Inkubationsdauer von $60 \mathrm{~min}$ erreicht. Als Ausdruck einer Sedimentation der Immunkomplexe beginnt das Streulicht bei hohen Fibronectinkonzentrationen in der Küvette $(9,6 \mathrm{mg} / \mathrm{l}) 75 \mathrm{~min}$ nach Beginn der Inkubation wieder zu fallen, während bei niedrigeren, im physiologischen Bereich liegenden Konzentrationen $(2,98 \mathrm{mg} / \mathrm{l})$ die Spannung bis mindestens 120 min stabil ist. Verlängerungen der üblichen Inkubationszeit von $60 \mathrm{~min}$ sind somit ohne Einfluß auf den Meßwert, wohingegen bei einer Inkubationsdauer von $45 \mathrm{~min}$ durchschnittlich um

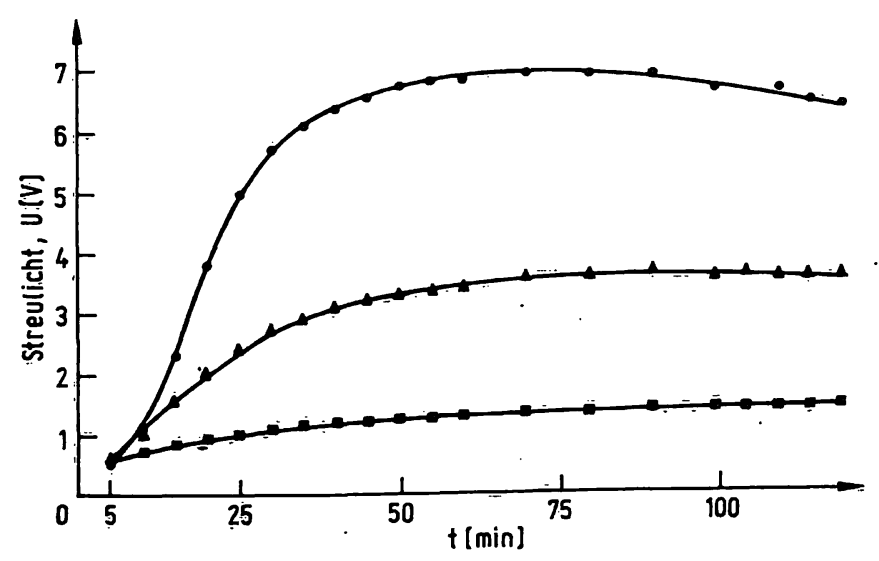

Abb. 1. Kinetik der Immunkomplexbildung zwischen PlasmaFibronectin und Antiserum.

Protein-Standardplasma wurde in den Verdünnungen $1: 13,1: 42$ und $1: 130$ eingesetzt, so daß Fibronectinkonzentrationen von $9,6 \mathrm{mg} / 1(\bullet-\bullet), 2,97 \mathrm{mg} / 1(\Delta-\Delta)$ und $0,96 \mathrm{mg} / 1\left(\square_{-}\right)$im Inkubationsansatz resultierten.
$10 \%$ niedrigere Streulichtsignale registriert werden (Abb. 1). Bei Verwendung von Dulbecco's phosphatgepufferter Lösung anstelle von ungepufferter physiologischer $\mathrm{NaCl}$-Lösung zur Plasmaverdünnung werden reduzierte Streulichtsignale erhalten, wobei das Ausmaß der Erniedrigung abhängig ist von der Antigenkonzentration in der Küvette und von der Inkubationsdauer (Abb. 2). So liegen nach $120 \mathrm{~min}$ bei einer Konzentration von $2,97 \mathrm{mg} / \mathrm{l}$ Fibronectin die Meßwerte mit phosphatgepufferter Salzlösung etwa um $15 \%$ unter den mit $0,154 \mathrm{~mol} / 1 \mathrm{NaCl}$ erhaltenen. Der Abbildung 2 ist weiterhin zu entnehmen, daß der Zusatz von Polyethylenglykol 6000 in einer Konzentration von $20 \mathrm{~g} / 1$ Küvetteninhalt in beiden Arten von Inkubationsmedium zu einer deutlichen Erhöhung des Streulichtes führt (bei $2,97 \mathrm{mg} / 1$ Fibronectin und einer Inkubationsdauer von 60 min um 53\%), wohingegen die Kinetik der Immunkomplexbildung nahezu unbeeinflußt bleibt.

Die Intensität des Streulichtes verändert sich in Abhängigkeit von der eingesetzten Antigenmenge in einer für Heidelberger-Kurven typischen Weise (Abb. 3). Mit Verlängerung der Inkubationsdauer von $15 \mathrm{~min}$ auf 120 min kommt es bei sehr niedrigen Antigenkonzentrationen in der Küvette (z.B. 0,7 mg/l) zu einer 100\%igen Erhöhung des Streulichtes und damit zu einer Anhebung der unteren Nachweisgrenze. Mit zunehmender Inkubationsdauer wird außerdem eine Vergrößerung des Meßbereiches durch Verschiebung des Äquivalenzpunktes zu höheren Antigenkonzentrationen (von $10 \mathrm{mg} / 1$ bei 15 minutiger $\mathrm{zu} 18 \mathrm{mg} / \mathrm{l}$ bei 60 minutiger Inkubation) erreicht (Abb. 3). Unter Berücksichtigung einer Mindestfüllmenge der Küvette von $150 \mu \mathrm{l}$ ist es möglich, lasernephelometrisch 1,2 mg Fibronectin pro 1 Körperflüssigkeit (entsprechend $90 \mathrm{ng}$ Antigen in der Küvette) zuverlässig nachzuweisen, eine Empfindlichkeit, die durch Zusatz von Polyethylenglykol (siehe oben) noch $\mathrm{zu}$ steigern ist.

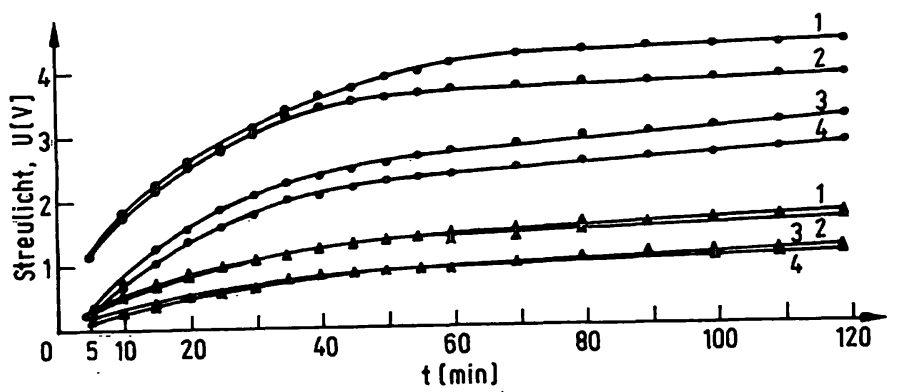

Abb. 2. Kinetik der Immunkomplexbildung zwischen Fibronectin und Antiserum in Abhängigkeit von der Art der Verdünnungslösung des Plasmas mit und ohne Polyethylenglykol.

Protein-Standardplasma wurde wie in Abbildung 1 beschrieben verdünnt, um Fibronectinkonzentrationen in der Küvette von $2,97 \mathrm{mg} / 1(\bullet-\bullet)$ und $0,96 \mathrm{mg} / 1(\Delta-\Delta)$ zu erhalten. Die Verdünnung erfolgte entweder mit $0,154 \mathrm{~mol} / 1 \mathrm{NaCl}(1,3)$ oder mit phosphatgepufferter NaCl-Lösung $(2,4)$, die entweder $20 \mathrm{~g} / 1(1,2)$ oder kein $(3,4)$ Polyethy lenglykol enthielten. 


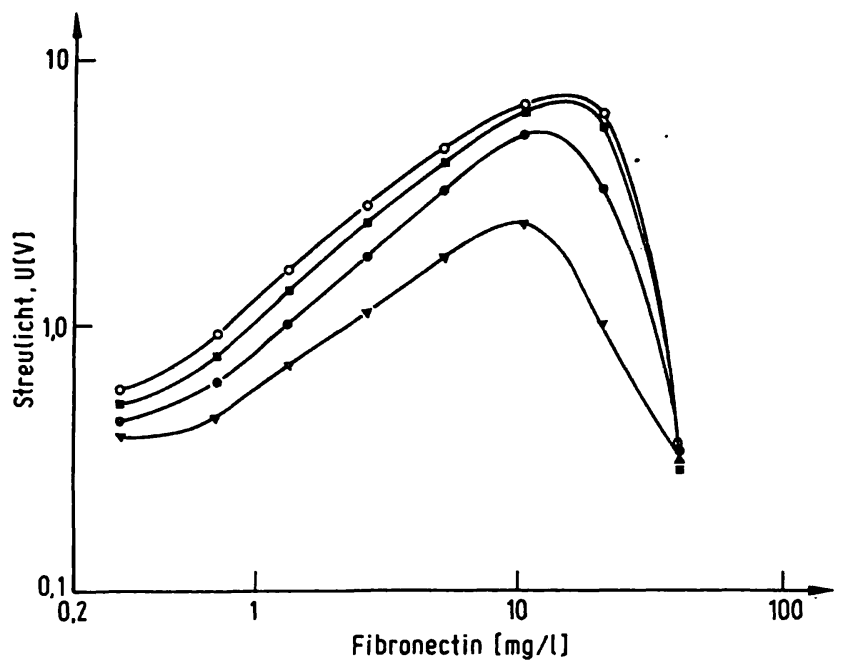

Abb. 3. Immunkomplexbildung zwischen Fibronectin und Antiserum in Abhängigkeit von der Antigenkonzentration in der Küvette.

Die Streulichtmessung erfolgte nach einer Inkubationszeit von $15 \mathrm{~min}(\Delta-\Delta), 30 \mathrm{~min}(\bullet-\bullet), 60 \mathrm{~min}(--)$ und $120 \mathrm{~min}(0-0)$.

Eine typische Referenzkurve für einen Antigenbereich von $0,6-9,6 \mathrm{mg} / \mathrm{l}$ Küvetteninhalt, der etwa die untere Hälfte des aufsteigenden Teiles der Heidelberger-Kurve repräsentiert, zeigt Abbildung 4. 95\% der für ein gesundes Referenzkollektiv ermittelten Streulichtsignale für Fibronectin im Plasma kommen auf den unteren 40\% der Referenzkurve zu liegen. Damit ist gewährleistet, daß auch bei stark erhöhten pathologischen Fibronectinkonzentrationen im Plasma bzw. bei hohen Antigen-

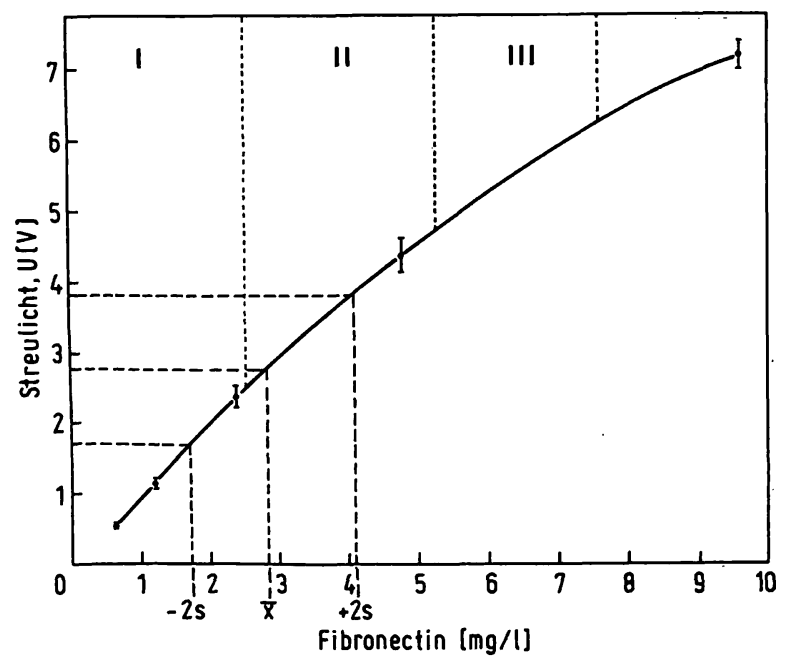

Abb. 4. Referenzkurve für Plasma-Fibronectin. Protein-Standardplasma wurde in den Verdünnungen $1: 13,1: 26,1: 52,1: 104$ und 1:208 mit $100 \mu 1$ Antiserum für $60 \mathrm{~min}$ inkubiert. Mittelwert $\pm 2 \mathrm{~s}$. Bereich der Streulichtsignale und Fibronectinkonżentrationen in der Küvette für ein Kollektiv von 110 gesunden Kontrollpersonen sind angegeben.

Die Abschnitte I bis III markieren die Bereiche der Standardkurve, für die zum Zwecke der rechner-unterstützten Auswertung Regressionsgeraden berechnet wurden. konzentrationen in anderen Körperflüssigkeiten bei unveränderter Vorverdünnung (1:51) stets im Antikörperexzeß gemessen wird. Die zur Ermittlung der Referenzkurve hergestellten Plasmaverdünnungen sind über einen Zeitraum von mindestens 5 Tagen stabil.

Uber die bei der lasernephelometrischen Bestimmung von Fibronectin im Plasma erreichten Präzisionen informiert Tabelle 1. Zur Durchführung von Präzisionskontrollen sind grundsätzlich bei $-20^{\circ} \mathrm{C}$ aufbewahrte Aliquots eines Humanplasmapools geeignet, doch können alternativ dazu einige der in Tabelle 2 untersuchten Kontrollseren herangezogen werden. Da ihre Fibronectinkonzentrationen (mit Ausnahme von Standardhumanplasma) im allgemeinen wesentlich niedriger als im Humanplasma sind, können nur durch entsprechend geringere Vorverdünnungen dem 1:51 verdünnten Humanplasma vergleichbare Streulichtsignale erhalten werden. Eine größere Anzahl von Kontrollseren scheint jedoch (nahezu) kein Fibronectin żu enthalten (Tab. 2).

Die Wiederfindungsrate des Antigens in einer kommerziell erhältlichen Lösung von Humanfibronectin beträgt $52 \%$, wenn die vom Hersteller angegebene Antigenkonzentration zugrunde gelegt wird.

Die Ergebnisse eines statistischen Vergleichs zwischen Lasernephelometrie und radialer Immundiffusion sind in Abbildung 5 zusammengefaßt. Die Präzisionen der Fibronectinbestimmung $(\mathrm{n}=14)$ mittels radialer Immundiffusion sind gekennzeichnet durch einen Variationskoeffizient von $2,4 \%$ (in der Serie) und 5,5\% (von Tag zu Tag) bei einer Antigenkonzentration von $125 \mathrm{mg} / \mathrm{l}$. Auch mit dieser Methode beträgt die Wiederfindung in der oben genannten Fibronectinlösung nur 52\%.

Die Art der Probenverwahrung (bei Raumtemperatur, $+4^{\circ} \mathrm{C},-20^{\circ} \mathrm{C}$ ) ist über einen Zeitraum von mindestens 10 Tagen ohne signifikanten Einfluß auf die gemessene Fibronectinkonzentration. Lagerung der Proben bei $-20^{\circ} \mathrm{C}$ über 6 Monate verändert den Meßwert nicht. In Ubereinstimmung mit früheren Befunden $(41,42)$ ergeben sich im Serum durchschnittlich um $18 \%$ niedrigere Fibronectinkonzentrationen als im EDTA-Plasma

Tab. 1. Ergebnisse der Präzisionskontrolle bei der Bestimmung der Fibronectin-Konzentration im Plasma mittels Lasernephelometrie.

\begin{tabular}{lccccccc}
\hline & \multicolumn{2}{c}{$\begin{array}{l}\text { Präzision } \\
\text { in der Serie }\end{array}$} & \multicolumn{5}{c}{$\begin{array}{l}\text { Präzision } \\
\text { von Tag zu Tag }\end{array}$} \\
\hline $\begin{array}{l}\text { Analysenzahl } \\
\begin{array}{l}\text { Mittelwert } \\
\text { (mg/l) }\end{array}\end{array}$ & 20 & 20 & 20 & 20 & 20 & 15 \\
$\begin{array}{l}\text { Standard- } \\
\text { abweichung } \\
\text { (mg/l) }\end{array}$ & 457 & 243 & 124 & 478 & 245 & 125 \\
$\begin{array}{l}\text { Variations- } \\
\text { koeffizient } \\
(\%)\end{array}$ & 8,9 & 3,9 & 3,3 & 18,8 & 14,7 & 18,8 \\
\hline & 2,1 & 1,6 & 2,6 & 3,9 & 6,0 & 7,0 \\
\hline
\end{tabular}


Tab. 2. Streulichtintensitäten und Fibronectinkonzentrationen in einigen Qualitätskontrollseren im Vergleich zu Humanplasma und Antiserum-NaCl-Gemisch (Volumina, 1 + 1). Die Vorverdünnungen waren für die Kontrollseren 1:21, für Humanplasma 1:51.

\begin{tabular}{|c|c|c|c|}
\hline $\begin{array}{l}\text { Kontrollscren } \\
\text { (Hersteller) }\end{array}$ & Charge & $\begin{array}{l}\text { Streulichtintensität } \\
\text { (V) }\end{array}$ & $\begin{array}{l}\text { immunreaktives } \\
\text { Fibronectin } \\
(\mathrm{mg} / \mathrm{l})\end{array}$ \\
\hline $\begin{array}{l}\text { Humanes Plasma }(\bar{x} \pm 2 s) \\
\text { Antiserum- } \mathrm{NaCl}\end{array}$ & - & $\begin{array}{l}1,4-2,50-3,3 \\
0,20\end{array}$ & $180-290-400$ \\
\hline $\begin{array}{l}\text { Standardhumanplasma (Behring) } \\
\text { Moni-Trol I-E (Dade) } \\
\text { Hyland-Q Pak (Hyland) } \\
\text { Seroquant A (Behring) } \\
\text { Hyland P (Hyland) } \\
\text { Hyland N (Hyland) } \\
\text { Moni-Trol II-E (Dade) } \\
\text { Normosic (Asid) } \\
\text { Validate A (Goedecke) } \\
\text { PKR (Asid) }\end{array}$ & $\begin{array}{l}502533 \mathrm{~A} \\
\text { LTD } 147 \mathrm{~B} \\
3656 \mathrm{P} 001 \mathrm{AAF} \\
624103 \\
\text { P } 11 \mathrm{E} \\
1 \mathrm{~N} 05 \mathrm{~A} \\
\text { PTD } 60 \mathrm{~A} \\
414 \mathrm{~B} \\
641117 \\
402\end{array}$ & $\begin{array}{l}4,22 \\
3,55 \\
2,96 \\
2,68 \\
2,58 \\
2,57 \\
2,40 \\
2,17 \\
1,95 \\
1,58\end{array}$ & $\begin{array}{r}234 \\
190 \\
152 \\
133 \\
127 \\
126 \\
116 \\
105 \\
94 \\
76\end{array}$ \\
\hline $\begin{array}{l}\text { Seronorm (Nyegaard) } \\
\text { Precilip (Boehringer) } \\
\text { Precinorm U (Boehringer) } \\
\text { Seronorm Lipid (Nyegaard) } \\
\text { Ortho (Ortho Diagnostics) } \\
\text { Ortho Abnormal (Ortho Diagnostics) } \\
\text { Ortho Ria Control I (Ortho Diagnostics) } \\
\text { Ortho Ria Control II (Ortho Diagnostics) } \\
\text { Pathonorm L (Nyegaard) } \\
\text { Bilirubin Control (Dade) } \\
\text { Precipath E (Bochringer) }\end{array}$ & $\begin{array}{l}147 \\
661 \\
839 \\
\text { S4 } \\
\text { S R } 215 \\
\text { 9S } 317 \\
3 \text { S } 407 \\
2 \text { S } 507 \\
12 \\
\text { B;C } 941 \\
833\end{array}$ & $\begin{array}{l}1,54 \\
1,37 \\
1,36 \\
0,74 \\
0,72 \\
0,63 \\
0,51 \\
0,43 \\
0,18 \\
0,17 \\
0,14\end{array}$ & $\begin{array}{l}74 \\
65 \\
65 \\
34 \\
33 \\
29 \\
23 \\
18,8 \\
- \\
-\end{array}$ \\
\hline
\end{tabular}

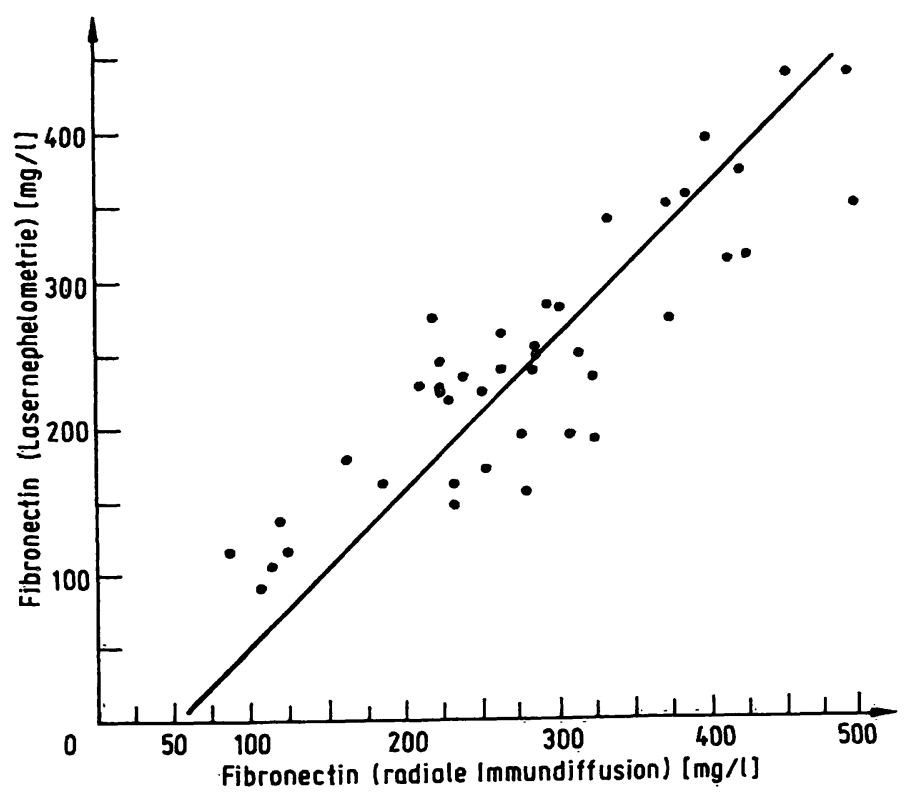

Abb. 5. Statistischer Vergleich der mit der Lasernephelometrie und radialen Immundiffusion ermittelten Fibronectinkonzentrationen in 42 Patientenplasmen. $\mathrm{n} \equiv 42 ; \mathrm{y}=1,0434 \mathrm{x}-54,59 ; \mathrm{r}=0,859$

(Tab. 3). Die mit Heparin antikoagulierten Plasmen weisen im allgemeinen niedrigere und stark streuende $\mathrm{Fi}$ bronectinkonzentrationen auf. Eine lasernephelometrische nachweisbare Inhibition der Bildung des Fibronectin-Antikörper-Komplexes durch Heparin konnte bis zu einẹ Polyanionenkonzentration von $90 \mathrm{mg} / \mathrm{l}$ Küvetten-
Tab. 3. Vergleich der lasernephelometrisch ermittelten Fibronectin-Konzentrationen im EDTA-Plasma, HeparinPlasma und Serum von 12 gesunden Probanden.

Von $10 \mathrm{ml}$ venös entnommenen Blutes wurden je $3 \mathrm{ml}$ in EDTA-beschich teten Blutbildröhrchen (Fa. Sarstedt) oder Heparin-enthaltenden Gefäßen (Fa. Sarstedt) antikoaguliert. Der Rest wurde bis zur vollständigen Gerinnung 1 Stunde bei Raumtemperatur aufbewahrt. Die Fibronectinbestimmung erfolgte im Zentrifugationsüberstand. Die Berechnung der Signifikanzen ( $\alpha$ ) erfolgte mit dem t-Test für verbundene Stichproben.

\begin{tabular}{|c|c|c|c|}
\hline & Heparin-Plasma & EDTA-Plasma & Serum \\
\hline $\begin{array}{l}\text { Mittelwert } \\
\text { (mg/l) }\end{array}$ & ${ }_{\alpha}^{178}=0,001$ & ${ }_{\alpha}^{306}=0,001$ & 245 \\
\hline $\begin{array}{l}\text { Standard- } \\
\text { abweichung } \\
\text { (mg/l) }\end{array}$ & 71 & 45 & 38 \\
\hline $\begin{array}{l}\text { Variations- } \\
\text { koeffizient } \\
(\%)\end{array}$ & 40,0 & 14,7 & 15,5 \\
\hline
\end{tabular}

inhalt nicht festgestellt werden. Bei einer Heparinkonzentration von $170 \mathrm{mg} / \mathrm{l}$ beträgt die Wiederfindung des Fibronectins noch $83 \%$.

Als Grundlage der rechner-assistierten Auswertung der Streulichtintensitäten (Spannungen) dient die in Abbildung 4 als Beispiel dargestellte Referenzkurve. Da auf Grund ihres flachen Verlaufes mit einer Exponentialfunktion keine optimale Kurvenanpassung erreichbar 
war, wurde die Kurve in 3 Bereiche aufgeteilt, für die die Regressionsgeraden ( $x=$ Spannung, $y=$ Fibronectinkonzentration) $y=0,975 x+0,0598$ (I), $y=1,18 x-$ 0,467 (II) und $y=1,549 x-2,258$ (III) berechnet wurden (Abb. 4). In allen drei Teilbereichen betragen die Korrelationskoeffizienten zwischen wahrer und idealisierter Kurve 0.999. Unter Berücksichtigung der Probenverdünnung wurden die Funktionen in den Rechner HP 9815 A eingegeben, der somit Meßsignale (V) und Fibronectinkonzentrationen ausdruckte.

\section{Klinische Anwendungen}

Die lasernephelometrisch im Plasma bestimmte mittlere Fibronectinkonzentration für gesunde Männer im Altersbereich zwischen 20 und 45 Jahren beträgt $291 \mathrm{mg} / \mathrm{l}$, Frauen weisen eine signifikant niedrigere Konzentration von $259 \mathrm{mg} / \mathrm{l}$ auf (Tab. 4). Die Verteilungsfunktion konnte nich eindeutig bestimmt werden.

Im Rattenplasma $(n=10)$ beträgt die mit Anti-Humanfibronectin gemessene immunreaktive Konzentration $(\bar{x} \pm \mathrm{s})$ des "cold-insoluble globulin" $228 \pm 11 \mathrm{mg} / \mathrm{l}$.

Stichprobenuntersuchungen ergaben für die Gruppe der Mammacarcinome $(n=7)$ und Carcinome des weiblichen Genitaltraktes (Corpus-, Collum-, Ovarialcarcinome) sowie bei Patientinnen mit Morbus Crohn signifikant erhöhte Fibronectinkonzentrationen im Plasma, wohingegen derartige Unterschiede bei Patienten mit Carcinomen des Verdauungstraktes (Ösophagus, Magen, Rektum) und mit Bronchialcarcinomen $(n=4)$ nicht nachweisbar waren (Tab. 4). Mit Ausnahme erhöhter Konzentrationen (400-500 $\mathrm{mg} / \mathrm{l}$ ) bei einigen Immuno-

Tab. 4. Fibronectinkonzentrationen im Plasma gesunder Personen und Patienten mit Carcinomen und Morbus Crohn. Die Referenzbereiche wurden mit dem nicht-parametrischen Verfahren nach Murphy (39) ermittelt; die Bereiche, die $80 \%$ der Meßwerte mit $\alpha=0,1$ umfassen und die Mittelwerte sind angegeben.

Für pathologische Plasmen sind die Streubereiche und die mittleren Konzentrationen aufgeführt. Die Signifikanzen wurrden mit dem t-Test für unverbundene Stichproben berechnet.

\begin{tabular}{llll}
\hline Herkunft des Plasmas & Anzahl & Fibronectin & $\begin{array}{l}\text { Signifi- } \\
\text { kanzen }\end{array}$ \\
& & $(\mathrm{mg} / \mathrm{l})$ & $(\alpha)$ \\
\hline
\end{tabular}

\section{Gesunde}

männliche Erwachsene $101 \quad 229-291-379$

Gesunde

weibliche Erwachsene $\quad 39 \quad 196-259-360$

0,01

Carcinome des weiblichen

Genitaltraktes und

Mammacarcinome

13

$253-332-412$

0,001

Carcinome des

Verdauungs- und

Respirationstraktes (Männer)

Ileitis terminalis Crohn

(Frauen) cytompatienten ergaben sich bei einer Vielzahl anderer Erkrankungen keine signifikanten Abweichungen vom Referenzwert.

Bei Synovialflüssigkeiten $(n=40)$, die nach zytologischen, klinisch-chemischen und physikochemischen Kriterien $(43,44)$ in nicht-entzuindliche und entzündliche Ergüsse getrennt wurden, $k$ konnte in der letztgenannten Gruppe eine signifikante Zunahme der Fibronectinkonzentration festgestellt werden (Tab. 5). In nicht-entzündlichen Punktionsflüssigkeiten beträgt die mittlere Antigenkonzentration etwa nur 50\% der Konzentration im Plasma. Zwischen Gesamtproteinund Fibronectingehalt besteht statistisch kein Zusammenhang.

Ähnlich den Veränderungen in den Synovialflüssigkeiten kommt es auch in entzündlichen Liquores cerebro-spinales żu Erhöhungen der Fibronectinkonzentrationen, deren Ausmaß stärker mit der Zellzahl $(r=0,898)$ als mit der Gesamtproteinkonzentration ( $r=0,797)$ korreliert (Tab. 6). Die extrem niedrigen Antigenkonzentrationen in gesunden und pathologischen Liquores machen eine 5:1 Konzentrierung vor der Inkubation mit dem Antiserum notwendig, um Streulichtintensitäten von mindestens $0,5 \mathrm{~V} \mathrm{zu}$ erhalten.

Dem Plasma vergleichbare Antigenbereiche konnten wir für die überwiegende Zạhl der untersuchten Seminalplasmen feststellen (Tab. 7). Die erhebliche Variabilität der individuellen seminalen Fibronectinkonżentrationen läßt statistisch keine Beziehung $(r=0,07)$ zur Spermatozoenzahl erkennen.

Sehr niedrige, zwischen 3,4 und 24,5 mg/1 (Mittelwert $16 \mathrm{mg} / \mathrm{l}$ ) sich bewegende Konzentrationen des immunreaktiven Fibronectins sind in den Amnionflüssigkeiten $(n=6)$ der 38. Schwangerschaftswoche nachweisbar, bei einer Probe aus der 18. Woche konnte jedoch eine

Tab. 5. Konzentrationen von Fibronectin und Gesamtprotein in Synovialflüssigkeiten nicht entzündlicher und entzündlicher Ätiologie.

Die Unterscheidung zwischen beiden Gruppen von Ergüssen erfolgte nach den früher aufgestellten klinischchemischen, zy tologischen und physikochemischen Kriterien $(43,44)$.

Die Signifikanzen der Unterschiede $(\alpha)$, geprïft mit dem Wilcoxon-Test; und die Korrelationen ( $\mathrm{r}$ ) zwișchen Fibronectin- und Proteinkonzentration sind angegeben (in Klammern).

\begin{tabular}{lccc}
\hline $\begin{array}{l}\text { Herkunft der } \\
\text { Synovialflüssigkeit }\end{array}$ & Anżahl & $\begin{array}{l}\text { Fibronectin } \\
(\mathrm{mg} / \mathrm{l})\end{array}$ & $\begin{array}{l}\text { Gesamt- } \\
\text { protein } \\
(\mathrm{g} / \mathrm{l})\end{array}$ \\
\hline & & \multicolumn{2}{c}{$(\mathrm{r}=0,37)$} \\
$\begin{array}{l}\text { Nicht-enzündlicher } \\
\text { Erguß }\end{array}$ & 23 & $153 \pm 65$. & $28,5 \pm 5,8$ \\
& & $(\alpha=0,001)$ & $\cdot(\alpha=0,001)$ \\
Entżüdlicher & &. & $47,9 \pm 5,2$ \\
Erguß & 17 & $318 \pm 129 \quad(\mathrm{r}=0,03)$ \\
\hline
\end{tabular}


Tab. 6. Fibronectin- und Gesamtproteinkonzentrationen sowie Zellzahl in normalen und pathologischen Liquores cerebrospinales.

Vor der Bestimmung wurde der Liquor 5:1 konzentriert. Die Zahl der untersuchten normalen Liquores ist in Klammern angegeben, für Gesamtprotein und Fibronectin sind Konzentrationsbereiche und Mittelwerte aufgeführt. Die Korrelationen ( $r$ ) wurden berechnet.

\begin{tabular}{lrll}
\hline & $\begin{array}{l}\text { Zellzahl } \\
\left(\frac{\left.\mu\right|^{-1}}{3}\right)\end{array}$ & $\begin{array}{l}\text { Fibronectin } \\
(\mathrm{mg} / \mathrm{l})\end{array}$ & $\begin{array}{l}\text { Gesamtprotein } \\
(\mathrm{g} / \mathrm{l})\end{array}$ \\
\hline $\begin{array}{l}\text { Normaler } \\
\text { Liquor (5) }\end{array}$ & $1-2$ & $0,22-0,41-0,56$ & $0,20-0,42-0,45$ \\
Pathologischer & 1 & 0,44 & \\
Liquor & 10 & 0,23 & 0,68 \\
& 778 & 0,51 & 0,30 \\
& 4200 & 0,57 & 0,62 \\
& 4200 & 1,06 & 1,51 \\
& 6100 & 1,36 & - \\
& 37800 & 2,18 & 0,46 \\
& $\mathrm{r}=0,898$ & $\mathrm{r}=0,797$ \\
\hline
\end{tabular}

Tab. 7. Fibronectinkonzentration im Seminalplasma und Spermienzahl im Ejakulat von 14 Patienten.

\begin{tabular}{ll}
\hline $\begin{array}{l}\text { Fibronectin } \\
(\mathrm{mg} / \mathrm{l})\end{array}$ & $\begin{array}{l}\text { Anzahl der Spermien } \\
\left(10^{9} / 1\right)\end{array}$ \\
\hline 330 & $-($ Vasektomie $)$ \\
297 & 21,5 \\
277 & $-($ Vasektomie $)$ \\
272 & 74,8 \\
246 & 4 \\
234 & $-($ Vasektomie $)$ \\
234 & 34 \\
196 & $-($ Vasektomie $)$ \\
184 & 86,4 \\
143 & 7,3 \\
143 & 4,5 \\
117 & 92 \\
100 & 0,5 \\
98 & 0 \\
\hline
\end{tabular}

Konzentration von $80 \mathrm{mg} / \mathrm{l}$ gemessen werden. Aufgrund dieser geringen Antigenkonzentration haben sich 1:4 Vorverdünnungen der Amnionflüssigkeiten für die Lasernephelometrie als optimal erwiesen.

\section{Diskussion}

Die vorgelegten Ërgebnisse zeigen, daß die methodischen Möglichkeiten zur Quantifizierung des ,cold-insoluble globulin" in biologischen Flüssigkeiten durch Anwendung der Lasernephelometrie bedeutungsvoll erweitert werden. Dạs Verfahren erweist sich als präzise, empfindlich, schnell, relativ einfàch, voll mechanisierbar und auf verschiedene menschliche und tierische Körperflüssigkeiten anwendbar. Präzision und Probendurchsatz kọnnen durch Adaptation einer automatischen Füllstation an das Nephelometer weiter gesteigert werden. Diese Merkmale, besonders die elektronische Auswertung der primären Meßwerte, lassen die lasernephelo- metrische Fibronectinbestimmung gegenüber den bisher angewandten immunologischen Verfahren $(1,15$, 27-29) als vorteilhaft erscheinen. Die mit ihr ermittelten Antigenkonzentrationen zeigen teilweise eine nur ungenügende Úbereinstimmung mit denen der radialen Immundiffusion (Abb. 5). Die von uns angegebenen Referenzbereiche für Fibronectiin im Plasma, die in Bestätigung früherer Befunde $(45,46)$ geschlechtsabhängig verschieden sind, stimmen jedoch gut mit der überwiegenden Zahl der früher mitgeteilten Werte $(41,42$, 47) überein.

Obwohl diese Angaben auf die Richtigkeit der Lasermethode hinweisen, muß es gegenwärtig dennoch als Nachteil angesehen werden, daß wegen Fehlens geeigneter Richtigkeitskontrollseren dieses Kriterium der analytischen Zuverlässigkeit nicht geprüft werden kann. Die Wiederfindung von nur 50\% der angegebenen Konzentration einer kommerziell erhältlichen reinen $\mathrm{Fi}$ bronectinlösung kann u.a. auf eine unrichtige Einwaage seitens des Herstellers, auf Veränderungen der Immunreaktivität während der Antigenlagerung und -präparation sowie auf Störungen der Immunkomplexbildung durch Komponenten des Puffersystems zurückzuführen sein. Zur Unterstützung dieser Gründe sei betont, daß die (scheinbar) reduzierte Wiederfindung sowohl bei der Lasernephelometrie als auch bei der radialen Immundiffusion feststellbar war und somit methodenunspezifisch ist.

Klinische, diagnostische und pathobiochemische Bedeutung des Fibronectins im Plasma sind gegenwärtig noch ungewiß. Es konnte nachgewiesen werden, daß zirkulierendes Fibronectin in die gleichen Zelloberflächenstrukturen inkorporiert werden kann wie das endogen synthetisierte Glykoprotein (48). Plasmafibronectin könnte somit zum Aufbau der perizellulären Matrix solcher Zellen dienen, die zur Synthese des LETS-Protein inkompetent sind (48). Obwohl bei einigen Erkrankungen erhöhte (49-51) und erniedrigte (41, 46, 50, 52-54) Konzentrationen nachgewiesen wurden, scheint die Fibronectinkonzentration im Blut, wie auch unsere Stichprobenuntersuchungen zeigen, auffällig konstant zu sein (45). Hingegen lassen sich signifikante Erhöhungen der Fibronectinkonzentrationen in den Synovialflüssigkeiten entzündlicher Gelenkerkrankungen feststellen. Eine Korrelation der Fibronectinkonzentration mit den ätiologischen Gruppen entzündlicher Gelenkerkrankungen läßt sich nicht angeben, möglicherweise besteht aber zwischen der Konzentration des Fibronectins und der entzündlichen Aktivität ein Zusammenhang. Dieses Protein kann somit in das Spektrum der Größen eingereiht werden, die zur klinisch-chemischen Differentialdiagnostik entzündlicher und nicht-entzündlicher Gelenkergüsse herangezogen werden $(43,44)$. Weitere Untersuchungen sind notwendig, um die Herkunft des synovialen Fibronectins (ortsständige Synthese oder Diafiltrat des Plasmas), seine mögliche molekulare Heterogenität (55) und die patho- 
biochemische Bedeutung erhöhter Fibronectinkonzentrationen im synovialen Entzündungsprozeß zu klären.

Erstmals konnten wir den Nachweis erbringen, daß Fibronectin im Seminalplasma in einer dem Blutplasma vergleichbaren Konzentration vorkommt. Auch hier müssen umfangreichere klinische Untersuchungen prüfen, ob die in Tabelle 7 festgestellten Variationen in den seminalen Fibronectinkonzentrationen von diagnostischer und/oder pathogenetischer Aussagekraft für Fertilitätsstörungen sind.

Im Gegensatz zu den bisher genannten Körperflüssigkeiten ist die immunreaktive Fibronectinkonzentration im Liquor cerebrospinalis und in der Amnionflüssigkeit sehr gering. Die lasernephelometrische Quantifizierung im Liquor gelingt nur nach Konzentrierung der Probe. Die von uns ermittelten Konzentrationen sind wesentlich niedriger als die bisher mitgeteilten, radioimmunologisch bestimmten Fibronectinkonzentrationen im Liquor cerebrospinalis (55a), was auf qualitative und/oder quantitative Unterschiede des Antigens in dem zur Kalibration verwendeten Standardplasma oder auf unterschiedliche Eigenschaften der Antikörperpräparationen zurückzuführen sein könnte.

Ein dem Plasmafibronectin immunologisch und strukturell sehr ähnliches, offenbar mit ihm aber nicht identisches Protein wurde in der Amnionflüssigkeit nachgewiesen $(56,57)$. Die in der Literatur angegebene Konzentration von $70 \mathrm{mg} / 1(56,57)$ ist, mit Ausnahme einer Probe aus der 18. Schwangerschaftswoche, höher als der lasernephelometrisch bestimmte Konzentrationsbereich, jedoch fehlen in früheren Mitteilungen eindeutige Angaben über den Zeitpunkt der Probennahme. Wie Zellkulturversuche (57) nachweisen konnten, entsteht das Amnionfibronectin nicht durch passive Ultrafiltration des Plasmas, sondern als Syntheseprodukt der als $\mathrm{E}$ (epithelial cells), AF (amniotic fluid cells) und F (fibroblastic cells) bezeichneten Zelltypen der Amnion-

\section{Literatur}

1. Kuusela, P., Ruoslahti, E., Engvall, E. \& Vaheri, A. (1976), Immunochemistry 13,639-642.

2. Ruoslahti, E., Vaheri, A., Kuusela, P. \& Linder, E. (1973), Biochim. Biophys. Acta 322, 352-358.

3. Gahmberg, C. G. \& Hakomori, S. (1973), Proc. Natl. Acad. Sci. USA 70, 3329-3333.

4. Yamada, K. \& Weston, J. (1974), Proc. Natl. Acad. Sci. USA 71, 3492-3496.

5. Hynes, R. O. \& Bye, J. M. (1974), Cell 3, 113-120.

6. Mosher, D. F. (1975), J. Biol. Chem. 250, 6614-6621.

7. Yamada, K. M. \& Olden, K. (1978), Nature 275, 179-184.

8. Grinnell, F. (1978), Int. Rev. Cytol. 53, 65-144.

9. Vaheri, A., Ruoslahti, E. \& Mosher, D. F. (1978), (Hrsg.) Fibroblast Surfache Protein, Ann. N. Y. Acad. Sci. 312.

10. Chen, A. B., Amrani, D. L. \& Mosesson, M. W. (1977), Biochim. Biophys. Acta 493, 310-322.

11. Ruoslahti, E. \& Vaheri, A. (1974), Nature 248, 789-791.

12. Engvall, E., Ruoslahti, E. \& Miller, E. J. (1978), J. Exp. Med. 147, 1584-1595.

13. Ruoslahti, E. \& Engvall, E. (1978), Ann. N. Y. Acad. Sci. $312,178-191$. flüssigkeit (58). Ob diesem Konstituenten der Amnionflüssigkeit ein physiologische Bedeutung zukommt, ist unbekannt.

Als Ausdruck der phylogenetisch sehr konservativen Struktur bestehen $z$ wischen den Fibronectinen verschiedener Tierspecies ausgedehnte immunologische Kreuzreaktivitäten $(1,13,59)$. Sie ermöglichen die Bestimmung der Konzentration von immunreaktivem Fibronectin in Kontrollseren tierischen Ursprungs. Jedoch muß betont werden, daß die derart ermittelte Konzentration wesentlich von der Stärke der immunologischen Kreuzreaktion beeinflußt wird und somit von der mit nicht-immunologischen Verfahren bestimmten Konzentration dieses Plasmaproteins in unterschiedlichem Ausmaße abweichen kann. Dennoch können sie, wie unsere Erfahrungen gezeigt haben, gegenwärtig aus Mangel geeigneter Kontrollplasmen zur Präzisionskontrolle herangezogen werden. Die immunologische Identität von Human- und Ratten-Plasmafibronectin (22) ausnutzend, kann die Lasernephelometrie zur Quantifizierung des Glykoproteins in diesem häufig verwendeten Versuchstier ebenso eingesetzt werden, wie zur quantitativen Bestimmung des LETS-Protein in bioptisch entnommenen Gewebsproben und zur Messung der Fibronectinproduktion durch in vitro kultivierte Zellen menschlicher oder tierischer Herkunft. Die Anwendung der lasernephelometrischen Bestimmung scheint somit geeignet, die pathobiochemische und diagnostische Bedeutung dieses möglicherweise sehr wichtigen Proteins im Plasma, aber auch in anderen Körperflüssigkeiten, durch Untersuchungen an einem umfangreichen Patientengut einer Klärung näher zu bringen.

\section{Danksagung}

Für die großzügige Bereitstellung des Fibronectin-Antiserums, des Standardplasmas und der Partigen-Platten für Fibronectin möchten wir Herrn Dr. F. Dati, Behring-Werke, Marburg/Lahn, vielmals danken.
14. Dessau, W., Adelmann, B. C., Timpl, R. \& Martin, G. R. (1978), Biochem. J. 169, 55-59.

15. Engvall, E. \& Ruoslahti, E. (1977), Int. J. Cancer 20, 1-5.

16. Balian, G., Click, E. M., Crouch, E., Davidson, J. M. \& Bornstein, P. (1979), J. Biol. Chem. 254, 1429-1432.

17. Ruoslahti, E. \& Vaheri, A. (1975), J. Exp. Med. 141, 497501.

18. Yamada, K. M., Olden, K. \& Pastan, I. (1978), Ann. N.Y. Acad. Sci. 312, 256-277.

19. Pearlstein, E. \& Gold, L. I. (1978), Ann. N.Y. Acad. Sci. 312, 278-292.

20. Vuento, M. (1979), Hoppe-Seyler's Z. Physiol. Chem. 360, $1327-1333$.

21. Yamada, K. M., Yamada, S. S. \& Pastan, I. (1975), Proc. 'Natl. Acad. Sci. USA 72, 3158-3162.

22. Molnar, J., Gelder, F. B., Ming Zong Lai, Siefring, G. E., Credo, R. B. \& Lorand, L. (1979), Biochemistry 18, 3909-3916.

23. Kaplan, J. E., Molnar, J., Saba, T. M. \& Allen, C. (1976), J. Reticuloendothel. Soc. 20, 375-384. 
24. Wartiovaara, J., Leivo, I., Virtanen, I., Vaheri, A. \& Graham, C. F. (1978), Nature 272, 355-356.

25. Vaheri, A., Ruoslahti, E., Westermark, B. \& Ponten, J. (1976), J. Exp. Med. 143, 64-72.

26. Vaheri, A. \& Ruoslahti, E. (1974), Int. J. Cancer 13, 579586.

27. Mancini, G., Carbonara, A. O. \& Heremans, J. F. (1965), Immunochemistry 2, 235-254.

28. Laurell, C. B. (1966), Anal. Biochem. 15, 45-52.

29. Ruoslahti, E., Vuento, M. \& Engvall, E. (1978), Biochim. Biophys. Acta 534, 210-218.

30. Conrad, A., Schürmann, J., Kreutz, F. H. \& Sieber, A. (1978), J. Clin. Chem. Clin. Biochem. 16, 299-305.

31. Daigneault, R. \& Lemieux, D. (1978), Clin. Biochem. 11, 28-31.

32. Töpel, M. (1979), Lab.med. 3, 59-64.

33. Shulman, G. (1979), Clin. Biochem. 12, 123-125.

34. Sieber, A. \& Gross, J. (1975), Prot. Biol. Fluids 23, 295298.

35. van Wersch, J., Peuckert, M. \& Greiling, H. (1977), Adv. Exp. Med. Biol. 76A, 619-623.

36. Voigt, H. W. (1977), Laboratoriumsblätter 27, 168-172.

37. Sieber, A. (1977), Laboratorium sblätter 27, 109-118.

38. Weichselbaum, T. E. (1946), Am. J. Clin. Pathol. 16, 40-48.

39. Murphy, R. B. (1948), Ann. Math. Statistics 19, 581-589.

40. Sachs, L. (1972), Statistische Auswertungsmethoden, 3. Auflage, Springer Verlag, Berlin.

41. Matsuda, M., Yoshida, N., Aoki, N. \& Wakabayashi, K. (1978), Ann. N.Y. Acad. Sci. 312, 74-92.

42. Mosesson, M. W. \& Umfleet, R. A. (1970), J. Biol. Chem. 245, 5728-5736.

43. Greiling, H. \& Kleesiek, K. (1978), Internistische Welt 4, $121-130$
44. Greiling, H., Kleesiek, K. \& Stuhlsatz, H. W. (1979), in Synovialflüssigkeit und synoviales Milieu (Thumb, N., Kelner, Ä., Klein, G. \& Zeidler, H., Hrsg.) S. 42-54, Georg Thieme Verlag, Stuttgart.

45. Fyrand, O. \& Solum, N. O. (1976), Thrombosis Res. 9, 447-455.

46. Mosher, D. F. \& Williams, E. M. (1978), J. Lab. Clin. Med. 91, 729-735.

47. Stathakis, N. E. \& Mosesson, W. (1977), J. Clin. Invest. 60 , 855-865.

48. Hayman, E. G. \& Ruoslahti, E. (1979), J. Cell. Biol. 83, $255-259$.

49. Forkman, B., Ganrot, P. O., Gennser, G. \& Rannevik, G. (1972), Scand. J. Clin. Lab. Invest. 29, 89-96.

50. Bruhn, H. D. \& Heimburger, N. (1976), Haemostasis 5 , 189-192.

51. Hällen, J. \& Laurell, C.-B. (1972), Scand. J. Clin. Lab. Invest. 29, 97-103.

52. Ganrot, P. O. (1972), Scand. J. Clin. Lab. Invest. 29, 83-88.

53. Johansson, B. G., Kindmark, C.-O., Trell, E. Y. \& Wollheim, F. A. (1972), Scand. J. Clin. Lab. Invest. 29, 117-126.

54. Aronsen, K. F., Ekeland, G., Kindmark, C.-O. \& Laurell, C.-B. (1972), Scand. J. Clin. Lab. Invest. 29, 127-136.

55. Clemmensen, I. \& Andersen, R. B. (1979), Abstr. IXth Europ. Kongreß für Rheumatologie, S. 27.

55a. Kuusela, P., Vaheri, A., Palo, J. \& Ruoslahti, E. (1978), J. Lab. Clin. Med. 92, 595-601.

56. Chen, A. B., Mosesson, H. W. \& Solish, G. I. (1976), Amer. J. Obstet. Gynecol. 125, 958-961.

57. Crouch, E., Balian, G., Holbrook, K., Hoehn, H. \& Bornstein, P. (1978), Ann. N.Y. Acad. Sci. 312, 410-413.

58. Hoehn, H., Bryant, E. M., Karp, L. E. \& Martin, G. R. (1974), Pediat. Res. 8, 746-754.

59. Kuusela, P., Ruoslahti, E. \& Vaheri, A. (1975), Biochim. Biophys. Acta 379, 295-303.
Priv.-Doz. Dr. med. A. M. Gressner Abteilung Klinische Chemie und Pathobiochemie der Medizinischen Fakultät der RWTH Goethestraße 27-29 D-5100 Aachen 
